Case Report

\title{
Maternal Perception of Decreased Fetal Movement in One Twin: A Clue Leading to the Early Detection of Absent Variability due to Acute Twin-to-Twin Transfusion Syndrome
}

\author{
Hirotada Suzuki, ${ }^{1}$ Tomoyuki Kuwata, ${ }^{1}$ Akihide Ohkuchi, ${ }^{1}$ Yukari Yada, \\ Shigeki Matsubara, ${ }^{1}$ and Mitsuaki Suzuki ${ }^{1}$ \\ ${ }^{1}$ Department of Obstetrics and Gynecology, Jichi Medical University School of Medicine, 3311-1 Yakushiji, Shimotsuke-shi, \\ Tochigi 329-0498, Japan \\ ${ }^{2}$ Department of Pediatrics, Jichi Medical University School of Medicine, 3311-1 Yakushiji, Shimotsuke-shi, Tochigi 329-0498, Japan \\ Correspondence should be addressed to Akihide Ohkuchi; okuchi@jichi.ac.jp
}

Received 2 June 2013; Accepted 14 July 2013

Academic Editors: I. Kowalcek, I. MacKenzie, E. C. Nwosu, and R. Sharony

Copyright (c) 2013 Hirotada Suzuki et al. This is an open access article distributed under the Creative Commons Attribution License, which permits unrestricted use, distribution, and reproduction in any medium, provided the original work is properly cited.

\begin{abstract}
Decreased fetal movement (DFM) perceived by pregnant women sometimes indicates imminent fetal jeopardy. It is unknown whether this also holds true for twin pregnancy. A 27-year-old primiparous woman with monochorionic diamniotic (MD) pregnancy had a slight difference of amniotic fluid volume at $31^{2 / 7}$ weeks of gestation. DFM only in one twin at $31^{4 / 7}$ weeks of gestation prompted her to receive urgent consultation. Since cardiotocogram indicated absent variability of one twin, we performed Cesarean section. Male infants weighing $2060 \mathrm{~g}$ and $1578 \mathrm{~g}$ were delivered; hemoglobin was 20.7 versus $10.8 \mathrm{~g} / \mathrm{dL}$, respectively; cardiothoracic ratio was $70 \%$ versus $44 \%$, respectively, indicating acute twin-to-twin transfusion syndrome (TTTS). The recipient infant had heart failure, which was still observed at 1 month postpartum. In conclusion, maternal perception of DFM indicated imminent fetal death or jeopardy caused by acute TTTS, suggesting that education regarding DFM for women with twin pregnancy may be clinically important.
\end{abstract}

\section{Introduction}

Decreased fetal movement (DFM) perceived by the mother sometimes indicates imminent fetal death or jeopardy in singleton pregnancy [1-5]. Does this also hold true for twin pregnancy? There has been no report that maternal perception of DFM in twin pregnancy is associated with imminent fetal death or jeopardy. We here describe a woman with twin pregnancy in whom DFM of one twin prompted her to receive urgent consultation to us, resulting in early detection of absent variability caused by acute twin-to-twin transfusion syndrome (TTTS).

\section{Case Presentation}

A 27-year-old primiparous woman with monochorionic diamniotic (MD) twins conceived spontaneously, sought maternal check-up at 9 weeks of gestation in a primary care hospital, and was checked every two weeks. At $26^{3 / 7}$ weeks of gestation, she was referred to our tertiary center due to "Satogaeri bunben" (a traditional ritual (support system) for perinatal women in Japan; "Satogaeri" means returning to the original family town or house and "bunben" means delivery); and her pregnant course every two weeks was uneventful until $31^{2 / 7}$ weeks, when the MD twins with vertex (I: rightlower side)-vertex (II: left-upper side) presentation showed amniotic fluid pocket of 7.9 and $2.4 \mathrm{~cm}$, respectively, indicating the appearance of slight difference. Estimated fetal body weight in the first and second twins was $1683 \mathrm{~g}$ and $1531 \mathrm{~g}$, respectively, suggesting that there was not significant fetal body weight discordancy. Both twins showed reassuring fetal statuses and water-filled bladders. Although this condition did not meet the criteria of TTTS [6], we planned to admit her 7 days later to monitor the fetuses more closely because amniotic fluid imbalance emerged abruptly. We educated her regarding DFM as a sign of imminent fetal death or jeopardy; 
therefore, she deliberately started to perceive fetal movements of both twins everyday [7]. Fortunately, she could perceive fetal movement in a fetus-by-fetus manner; she could easily discern one fetal movement from the other fetal movement because she had always felt the two fetuses' movements in almost the same location. Thus, although we did not recommend "modified count to 10" method in a fetal movement count chart [7] due to twin pregnancy, she has been paying attention to fetal movements of both fetuses. Five days before the booking day, she perceived DFM only in the right twin (I), with the left cotwin (II) moving as usual. In addition, she felt frequent uterine contractions. These changes prompted her to receive urgent consultation to us. Then, cardiotocogram indicated absent variability in the right twin (I), while reassuring pattern in the left twin (II), with uterine contraction every one to three minutes (Figure 1). There were no ultrasonographic signs indicating placental abruption. Considering immature cervix and acute appearance of absent variability of one twin, we decided to perform Cesarean section under general anesthesia. The first twin (I) was male infant weighing $2060 \mathrm{~g}$, with Apgar score of 1 and 5 at 1 and 5 minutes, respectively; and blood gas analysis in the umbilical artery showed $\mathrm{pH}$ of 7.13 and base excess of -10.8 . The second twin (II) was male infant weighing $1578 \mathrm{~g}$, with Apgar score of 1 and 6 at 1 and 5 minutes, respectively; and blood gas analysis in the umbilical artery showed $\mathrm{pH}$ of 7.20 and base excess of -10.8 . Hemoglobin of the first and second twins 2 hours after delivery was 20.7 and $10.8 \mathrm{~g} / \mathrm{dL}$, respectively; brain natriuretic peptide of each twin 2 days after delivery was 1459 and $2027 \mathrm{pg} / \mathrm{mL}$ (normal range: $<18.4 \mathrm{pg} / \mathrm{mL}$ ), respectively; and cardiothoracic ratio of each twin was 70\% (Figure 2) and 44\%, respectively. The first twin showed high systolic blood pressure $(80-100 \mathrm{~mm} \mathrm{Hg})$ and polyuria $(4-6 \mathrm{~mL} / \mathrm{kg} /$ hour $)$ just after delivery; left ventricular dimension was thick, ejection fraction was $65 \%$, and mild tricuspid regurgitation and mitral regurgitation were observed; and he had generalized edema just after delivery, but body weight decreased 20\% during 8 days after delivery. In contrast, the second twin showed very low systolic blood pressure $(20-30 \mathrm{~mm} \mathrm{Hg})$ and collapsed inferior vena cava just after delivery, indicating hypovolemia; however, echocardiography did not show any significant findings. Then, we diagnosed this condition as acute TTTS in view of both pregnancy course and the fetal findings. While the first recipient-twin was responded by diuretics and had large amount of urination, intraventricular hemorrhage and ventriculomegaly occurred 2 days after delivery; the ventriculomegaly was still observed at 1 month postpartum. The second donor twin showed no sequelae at 1 month postpartum. Patient anonymity was preserved and informed consent for reporting was obtained from the parents.

\section{Discussion}

Although it is not yet determined whether recommending all pregnant women to count fetal movements (universal fetal movement counting) reduces the fetal death, obstetricians should ask a pregnant woman to consult the caregivers when she felt DFM [8]. This case dramatically illustrated that maternal perception of DFM may save fetal/infant lives in not only

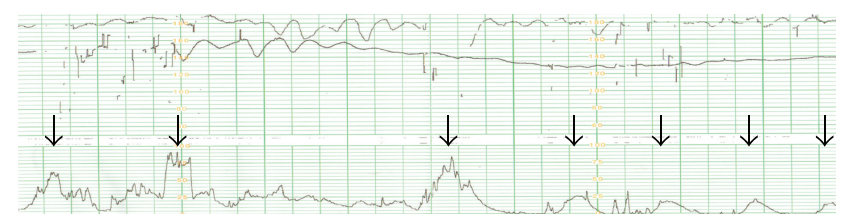

FIGURE 1: Cardiotocogram indicated absent variability in the right twin (I), and reassuring pattern in the other left twin (II), showing uterine contractions every one to three minutes. Arrows indicate uterine contractions.

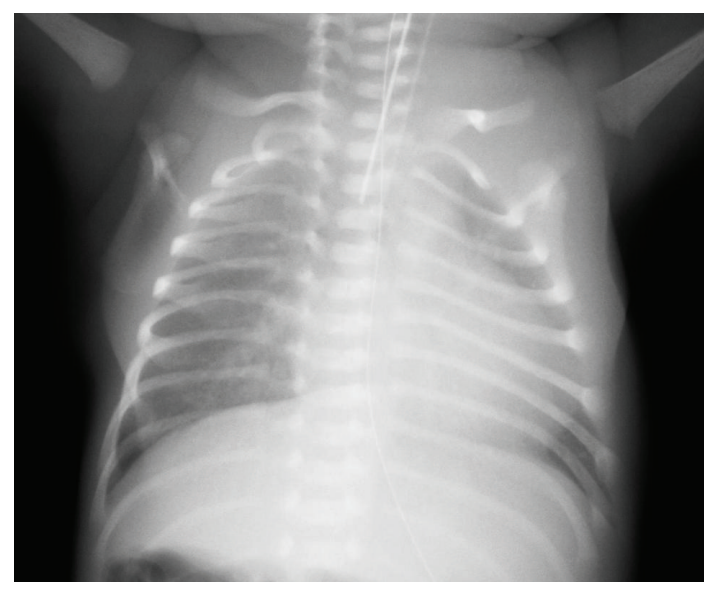

FIGURE 2: The chest X-ray of the first recipient-twin showed cardiac dilatation: the cardiothoracic ratio was $70 \%$.

singleton pregnancy but also in twin pregnancy, indicating the importance of educating pregnant women regarding DFM. To our knowledge, no previous reports showed possible effectiveness of educating pregnant women with twin pregnancy regarding DFM.

In this pregnant woman with MD twins, fetal movement in one twin acutely decreased and regular uterine contraction occurred at the same time. Although apparent placental vascular anastomoses were not determined by visual inspection in this case, previous reports clearly indicated that placenta in almost all MD twins has vascular anastomoses [9-11]. We do not know the reasons why DFM and frequent uterine contractions occurred simultaneously; however, uterine contraction may have increased the flow between the fetuses, which might have triggered, or might have led to, acute TTTS $[12,13]$.

It may have been more cautious if we admitted her at the time when amniotic fluid imbalance emerged abruptly at $31^{2 / 7}$ weeks of gestation, even though the criterion of TTTS was not fulfilled then. Van Mieghem et al. [14] showed that a fluid discordance of $\geq 3.1 \mathrm{~cm}$ diagnosed before 20 weeks selected the group at highest risk for TTTS. However, since a predictor of acute TTTS has not been identified yet, it is very difficult to predict acute TTTS, and we have not yet had a definite measure for it. Furthermore, we do not know if earlier admission might have led to earlier detection of occurrence of acute TTTS in our case with MD twins showing slight amniotic fluid imbalance after 26 weeks of gestation, and thus 
to earlier intervention before establishing heart failure in the first recipient twin.

When the fetal position in twin pregnancy frequently changes, the pregnant woman may feel difficulty in discriminating the movements in a fetus-by-fetus manner in other words, it may be very difficult for her to know one's decreased activity if only one twin becomes almost inactive. However, we believe that educating pregnant women with twin pregnancy regarding clinical meaning of DFM may be better encouraged even for women with twin pregnancy because we, from our experiences on twin pregnancy, know that not a few women with twin pregnancy could perceive fetal movements in a fetus-by-fetus manner. At least, letting them notice the importance of DFM may be far better than letting them unnoticed it.

We here describe the maternal perception of DFM in one twin indicating fetal jeopardy caused by acute TTTS. The maternal perception of DFM even in twin pregnancy may be associated with imminent fetal death. Educating pregnant women with twin pregnancy regarding clinical meaning of DFM may be clinically important for saving fetal/infant lives.

\section{Disclosure}

None of the authors have a financial or proprietary interest in any material or method mentioned in this paper.

\section{Acknowledgment}

The authors thank for Dr. Yume Suzuki, Pediatrician, who performed echocardiography for both twins and cooperated in writing the paper and discussion.

\section{References}

[1] J. F. Pearson and J. B. Weaver, "Fetal activity and fetal wellbeing: an evaluation," British Medical Journal, vol. 1, no. 6021, pp. 13051307, 1976.

[2] L. R. Leader, P. Baillie, and D. J. van Schalkwyk, "Fetal movements and fetal outcome: a prospective study," Obstetrics and Gynecology, vol. 57, no. 4, pp. 431-436, 1981.

[3] J. E. Whitty, D. A. Garfinkel, and M. Y. Divon, "Maternal perception of decreased fetal movement as an indication for antepartum testing in a low-risk population," The American Journal of Obstetrics and Gynecology, vol. 165, no. 4, pp. 1084-1088, 1991.

[4] J. V. H. Tveit, E. Saastad, B. Stray-Pedersen et al., "Reduction of late stillbirth with the introduction of fetal movement information and guidelines: a clinical quality improvement," $B M C$ Pregnancy and Childbirth, vol. 9, article 32, 2009.

[5] T. Stacey, J. M. D. Thompson, E. A. Mitchell, A. Ekeroma, J. Zuccollo, and L. M. E. Mccowan, "Maternal perception of fetal activity and late stillbirth risk: findings from the Auckland stillbirth study," Birth, vol. 38, no. 4, pp. 311-316, 2011.

[6] R. A. Quintero, W. J. Morales, M. H. Allen, P. W. Bornick, P. K. Johnson, and M. Kruger, "Staging of twin-twin transfusion syndrome," Journal of Perinatology, vol. 19, no. 8, pp. 550-555, 1999.

[7] T. Kuwata, S. Matsubara, T. Ohkusa et al., "Establishing a reference value for the frequency of fetal movements using modified 'count to 10' method," Journal of Obstetrics and Gynaecology Research, vol. 34, no. 3, pp. 318-323, 2008.
[8] L. Mangesi and G. J. Hofmeyr, "Fetal movement counting for assessment of fetal wellbeing," Cochrane Database of Systematic Reviews, no. 1, Article ID CD004909, 2007.

[9] K. E. A. Hack, P. G. J. Nikkels, C. Koopman-Esseboom et al., "Placental characteristics of monochorionic diamniotic twin pregnancies in relation to perinatal outcome," Placenta, vol. 29, no. 11, pp. 976-981, 2008.

[10] L. Lewi, M. Cannie, I. Blickstein et al., "Placental sharing, birthweight discordance, and vascular anastomoses in monochorionic diamniotic twin placentas," The American Journal of Obstetrics and Gynecology, vol. 197, no. 6, pp. 581.el-581.e8, 2008.

[11] A. Ohkuchi, H. Minakami, H. Shiraishi, I. Suzuki, T. Ohki, and I. Sato, "Intrauterine death of one twin, with rescue of the other, in twin-twin transfusion syndrome," Ultrasound in Obstetrics and Gynecology, vol. 19, no. 3, pp. 293-296, 2002.

[12] Y. Takahashi, S. Iwagaki, Y. Nakagawa, I. Kawabata, and T. Tamaya, "Uterine contractions increase fetal heart preload," Ultrasound in Obstetrics and Gynecology, vol. 22, no. 1, pp. 5356, 2003.

[13] Y. Takahashi, S. Iwagaki, Y. Nakagawa, I. Kawabata, and T. Tamaya, "Uterine contractions might increase heart preload in the recipient fetus in early-onset twin-twin transfusion syndrome: an ultrasound assessment," Prenatal Diagnosis, vol. 24, no. 12, pp. 977-980, 2004.

[14] T. van Mieghem, E. Eixarch, L. Gucciardo et al., "Outcome prediction in monochorionic diamniotic twin pregnancies with moderately discordant amniotic fluid," Ultrasound in Obstetrics and Gynecology, vol. 37, no. 1, pp. 15-21, 2011. 


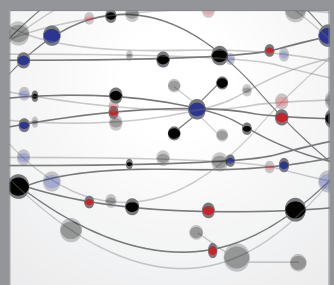

The Scientific World Journal
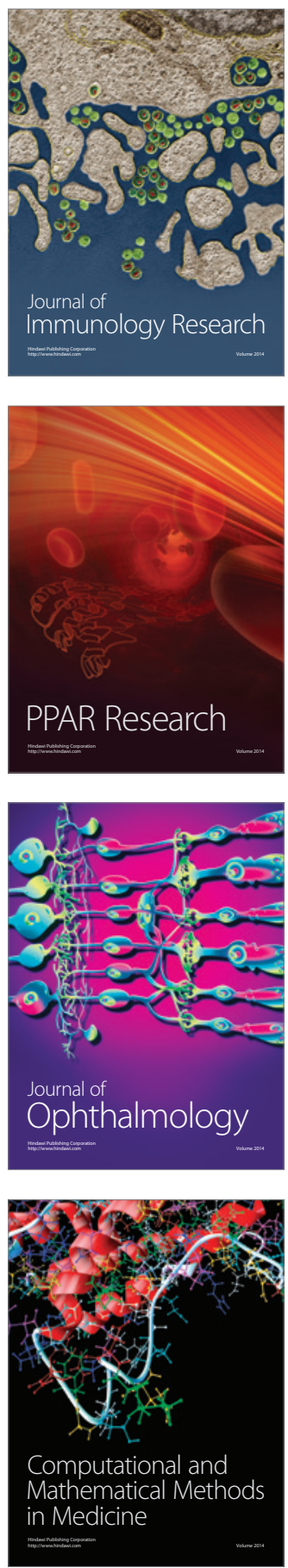

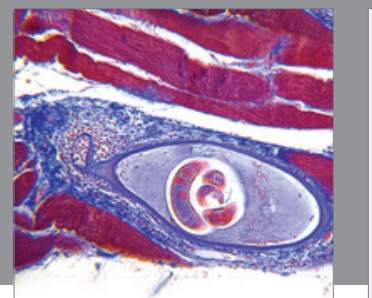

Gastroenterology

Research and Practice
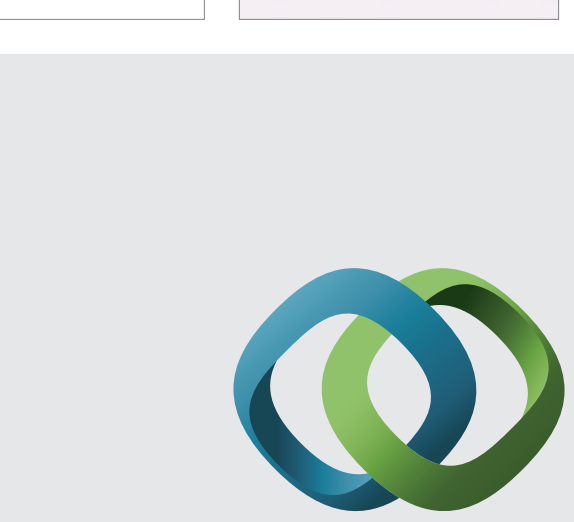

\section{Hindawi}

Submit your manuscripts at

http://www.hindawi.com
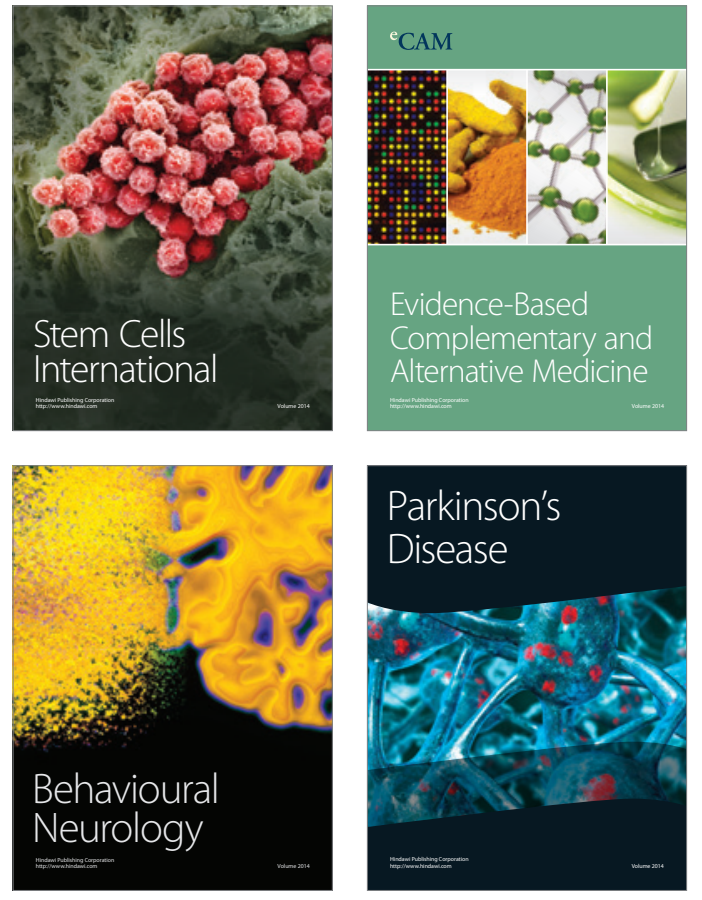
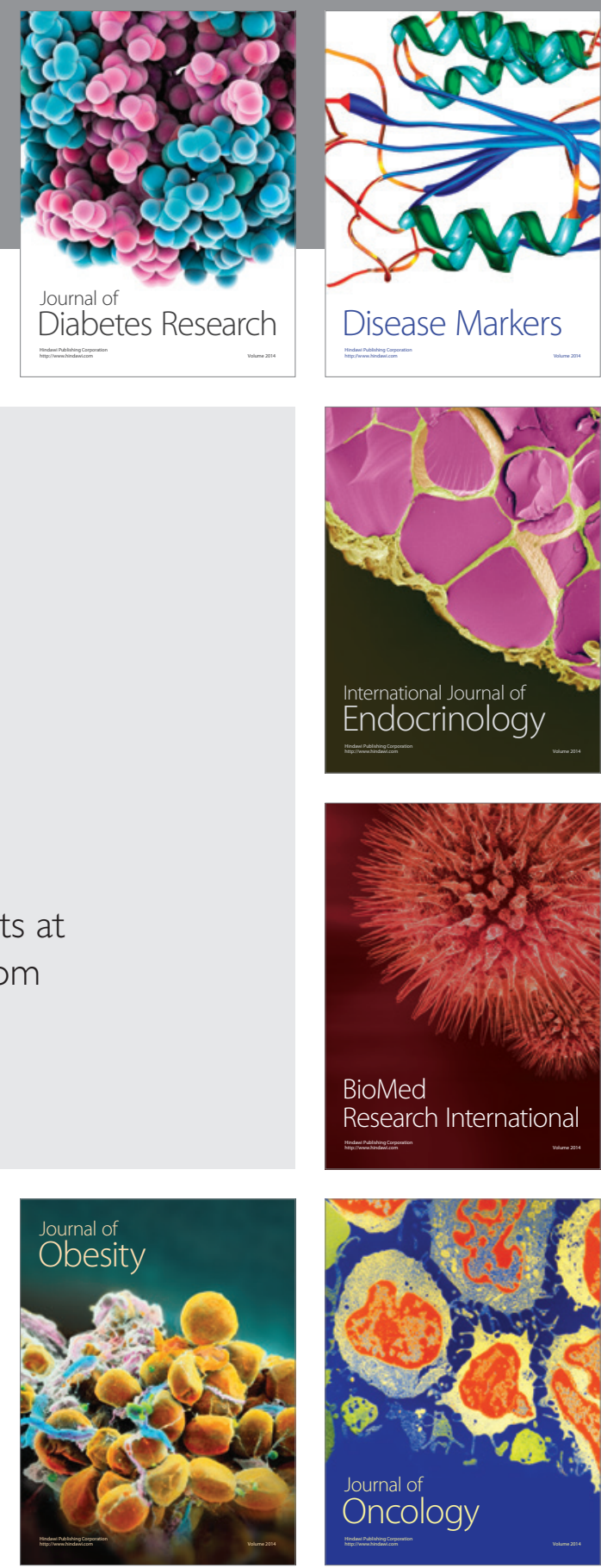

Disease Markers
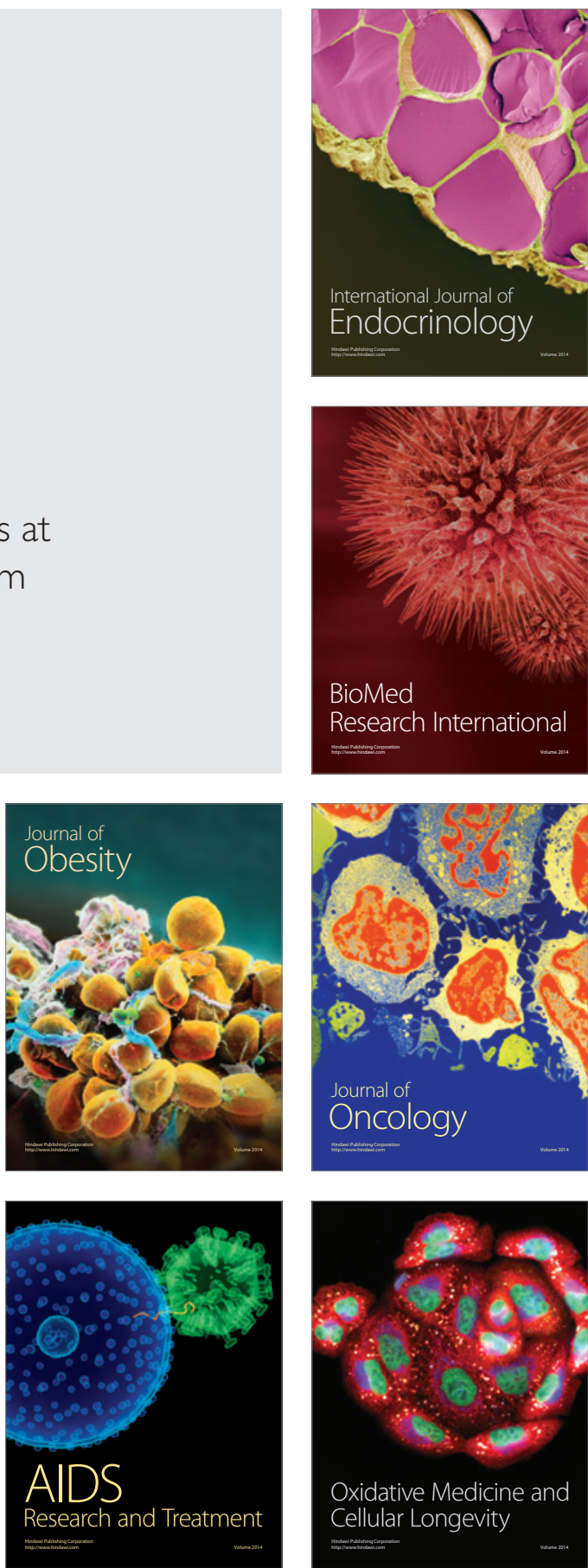